\title{
Hypercyclicity for the elements of the commutant of an operator
}

\author{
M. González \\ Department of Mathematics, University of Cantabria, Facultad de Ciencias, Avda. \\ de los Castros s/n, E-39071-Santander. Spain. \\ F. León-Saavedra \\ Department of Mathematics University of Cádiz Avda. de la Universidad s/n \\ 11405-Jerez de la Frontera. Spain.
}

\begin{abstract}
Given a bounded linear operator $T$ acting on a complex Banach space, we obtain a spectral condition implying that each non-scalar operator in the commutant of $T$ has a hypercyclic multiple, and we show several examples of operators satisfying this condition. We emphasize that for some of these examples we do not have a description of the commutant of $T$.
\end{abstract}

Key words: Hypercyclic operator, Hypercyclic commutant, Cesàro operator, Composition operator

PACS: Primary 47B37, Secondary 47B38, 47B99

\section{Introduction}

A bounded linear operator $T$ on a Banach space is called hypercyclic if it has a dense orbit. A vector $x$ for which the orbit $\left\{T^{n} x\right\}$ is dense is called a hypercyclic vector for $T$. We refer to the books [2] and [9] for information on hypercyclic operators.

\footnotetext{
Email addresses: manuel.gonzalez@unican.es (M. González), fernando.leon@uca.es (F. León-Saavedra).

1 The first author was supported by the Kingdom of Spain, Grant MTM2010-20190.

2 The second author was partially supported by Junta de Andalucía FQM-257, Vicerrectorado de investigación UCA.
} 
When we sometimes abusively say that an operator $T$ has a hypercyclic commutant, what we really mean is that each non-scalar operator in the commutant has a hypercyclic multiple (a non-scalar operator is an operator which is not a multiple of the identity). The study of operators with hypercyclic commutant was originated by Godefroy and Shapiro in their seminal 1991 paper [7]. Their work suggests that operators behaving like backward shifts tend to transfer hypercyclicity to "appropiate" operators in their commutant. The word "appropiated" means that we must discard from the commutant some operator that can never be hypercyclic, like the multiples of the identity or the contractions.

In their work, Godefroy and Shapiro use some descriptions of the commutants of the involved operators acting on Hilbert spaces which are not valid in the Banach space setting, and they also consider some bilateral weighted shifts and composition operators. Later, Herzog and Schmoeger [11] extended Godefroy and Shapiro's results to general operators $T$ on Banach spaces. Using the holomorphic functional calculus, they transfer the hypercyclicity to operators $\varphi(T)$ under certain spectral conditions on $T$. This work was improved by Bermúdez and Miller [2]. Since the holomorphic functional calculus does not cover the whole commutant, the following general question arises: Given a hypercyclic operator $T$, when $T$ has a hypercyclic commutant? Recently, V. Müller [13] improved the results of $[11,2]$, and discovered that these results become stronger if we consider operators with closed range. His study allows to prove that any non-scalar operator in the commutant of a generalized backward shift (acting on certain Banach spaces) is supercyclic, solving a longstanding question posed by Godefroy and Shapiro.

In this paper we study Banach space operators $T$ with hypercyclic commutant. To characterize the commutant of $T$ is a really complicated problem even for well known operators in Hilbert spaces. However we show that, under certain spectral conditions and without a description of the commutant of $T$, it is possible to ensure that $T$ has a hypercyclic conmutant.

The paper is structured as follows. In Section 2 we prove our main result (Theorem 2.1) that gives a sufficient spectral condition for an operator $T$ to have a "hypercyclic commutant". Section 3 is devoted to give examples of operators satisfying that spectral condition: Bilateral weighted shifts, unilateral weighted backward shifts, the adjoint of the discrete Cesàro operator acting on the sequence spaces $\ell^{p}$, the continuous Cesàro operator acting on $L_{p}(0,1)$, and the adjoint of the Cesàro operator acting on the Hardy spaces $H^{p}(\mathbb{D})$. We also show that some composition operators acting on the Hardy spaces, which are induced by linear fractional self-maps of the unit disk $\mathbb{D}$, fail both the hypothesis and the thesis of Theorem 2.1. The results of the paper suggest some questions which will be formulated in the last section. 


\section{Main Theorem}

In this section we give a general theorem for Banach space operators $T$ asserting that, under certain spectral conditions, each operator in the commutant of $T$ different from $\lambda I$ has a hypercyclic multiple.

Theorem 2.1 Let $T$ be a bounded operator on a (complex) Banach space $X$. Suppose that the spectrum of $T$ contains a non-empty connected open subset $U$ such that the following conditions are satisfied:

(1) Every $\lambda \in U$ is a simple eigenvalue of $T$,

(2) $\operatorname{span}\{\operatorname{Ker}(T-\lambda I): \lambda \in U\}$ is dense in $X$,

(3) there exists a holomorphic function $x: \lambda \in U \rightarrow \hat{x}(\lambda)=x_{\lambda} \in X$ such that $0 \neq x_{\lambda} \in \operatorname{ker}(T-\lambda I)$.

Then every operator in the commutant of $T$ different from $\lambda I$ has a hypercyclic multiple.

Proof. First let us see that given a non-empty open subset $V \subset U$, the set

$$
\operatorname{span}\{\operatorname{Ker}(T-\lambda I): \lambda \in V\}
$$

remains dense in $X$. Otherwise, there exists $0 \neq x^{\star} \in X^{\star}$ such that $x^{\star}(\hat{x}(\lambda))=$ 0 for all $\lambda \in V$. By the analyticity condition (3) we have that $x^{\star}(\hat{x}(\lambda))=0$ for all $\lambda \in U$, and by (1) and (2) $x^{\star}=0$, a contradiction.

Let $A \in L(X)$ in the commutant of $T$ with $A \neq \mu I$. For each $\lambda \in U$ we have $T x_{\lambda}=\lambda x_{\lambda}$, hence $T A x_{\lambda}=\lambda A x_{\lambda}$. Thus by condition (1) we have $A x_{\lambda}=a(\lambda) x_{\lambda}$ for some complex number $a(\lambda)$. So we get a function $a: U \rightarrow \mathbb{D}$. Boundedness of the operator $A$ provides boundedness of $a(\lambda)$ on $U$, and $A \neq \mu I$ implies that $a$ is non-constant. Let us see that $a: U \rightarrow \mathbb{D}$ is a holomorphic function.

For each $y^{\star} \in X^{\star}$, our function $a$ satisfies

$$
a(\lambda)=\frac{y^{\star}\left(A x_{\lambda}\right)}{y^{\star}\left(x_{\lambda}\right)} .
$$

Thus it is holomorphic on $U \backslash Z$, where $Z=\left\{\lambda: y^{\star}\left(x_{\lambda}\right)=0\right\}$ is a discrete subset of $U$. But for $\lambda_{0} \in Z$ we can take $u^{\star} \in X^{\star}$ such that $u^{\star}\left(x_{\lambda_{0}}\right) \neq 0$. This implies that our function

$$
a(\lambda)=\frac{u^{\star}\left(A x_{\lambda}\right)}{u^{\star}\left(x_{\lambda}\right)}
$$

is holomorphic also on $\lambda_{0}$, hence $a(\lambda)$ is holomorphic on $U$.

Since $a(\lambda)$ is non-constant, $a(U)$ is an open subset of the complex plane. Then for some $m \in \mathbb{C}$ the set $m \cdot a(U)$ intersects the unit circle. To prove that $m A$ 
is hypercyclic, let us see that the subsets

$$
\operatorname{span}\{\operatorname{ker}(\mu \mathrm{A}-\lambda \mathrm{I}):|\lambda|<1\} \text { and } \operatorname{span}\{\operatorname{ker}(\mu \mathrm{A}-\lambda \mathrm{I}):|\lambda|>1\}
$$

are dense in $X$, and the hypercyclicity of $m A$ will follow from the GodefroyShapiro Criterion (see Corollary 1.10 in [2]).

Indeed, $\operatorname{span}\{\operatorname{ker}(\mu \mathrm{A}-\lambda \mathrm{I}):|\lambda|<1\}=\operatorname{span}\left\{\operatorname{ker}(\mathrm{T}-\lambda \mathrm{I}): \lambda \in(m \cdot a)^{-1}(\mathbb{D})\right\}$ is dense by the considerations at the beginning of the proof, and a similar argument is valid for the other set.

Remarks 2.2 The hypothesis of Theorem 2.1 implies that

(a) the space $X$ is infinite dimensional and separable, and

(b) the operator $T$ fails the single valued extension property (see [1]).

\section{Some applications}

In this section we show that several concrete examples of operators satisfy the hypothesis of Theorem 2.1.

\subsection{Bilateral weighted shifts.}

Let us consider a bilateral weighted shift $W$ on the complex Hilbert space of sequences $\ell_{2}(\mathbb{Z})$, that is defined by

$$
W e_{n}=w_{n} e_{n+1},
$$

where $n$ runs through the set of all integers $\mathbb{Z},\left\{e_{n}: n \in \mathbb{Z}\right\}$ is the natural orthonormal basis of $\ell_{2}(\mathbb{Z})$, and $\left(w_{n}\right)$ is a bounded sequence of strictly positive numbers, the sequence of weights. We follow the notation used in the survey by Allen L. Shields [18].

We consider the quantities

$$
r_{3}^{+}(W):=\limsup _{n \rightarrow \infty}\left(w_{0} \cdots w_{n-1}\right)^{1 / n}
$$

and

$$
r_{2}^{-}(W):=\liminf _{n \rightarrow \infty}\left(w_{-1} \cdots w_{-n}\right)^{1 / n}
$$

It turns out (see [18] Theorem 9) that when $r_{3}^{+}(W)<r_{2}^{-}(W)$ the set

$$
U:=\left\{\lambda \in \mathbb{C}: r_{3}^{+}(W)<|\lambda|<r_{2}^{-}(W)\right\}
$$


is contained in the point spectrum of $W$, that every $\lambda \in U$ is a simple eigenvalue of $W$ and that a corresponding eigenvector is given by the following expression:

$$
x_{\lambda}=e_{0}+\sum_{n=1}^{\infty} \frac{w_{0} \cdots w_{n-1}}{\lambda^{n}} e_{n}+\sum_{n=0}^{\infty} \frac{\lambda^{n}}{w_{-1} \cdots w_{-n}} e_{-n} .
$$

Clearly the function $f(\lambda)=x_{\lambda}$ is analytic on $U$. Let us see that the set $\operatorname{span}\left\{\mathrm{x}_{\lambda}: \lambda \in \mathrm{U}\right\}$ is dense in $\ell_{2}(\mathbb{Z})$. Indeed, given $\left(y_{i}\right) \in \ell_{2}(\mathbb{Z})$ we have

$$
\left\langle x_{\lambda},\left(y_{i}\right)\right\rangle=y_{0}+\sum_{n=1}^{\infty} \frac{w_{0} \cdots w_{n-1}}{\lambda^{n}} y_{n}+\sum_{n=0}^{\infty} \frac{\lambda^{n}}{w_{-1} \cdots w_{-n}} y_{-n}
$$

Therefore if $\left\langle x_{\lambda},\left(y_{i}\right)\right\rangle=0$ for all $\lambda \in U$, then $y_{i}=0$ for all $i \in \mathbb{Z}$. Thus $W$ satisfies the conditions of Theorem 2.1 and we have the following result.

Theorem 3.1 Let $W$ be an injective bilateral shift with $r_{3}^{+}(W)<r_{2}^{-}(W)$. Then every operator in the commutant of $W$ different from $\lambda I$ has a hypercyclic multiple.

Remarks 3.2 It is not difficult to see that Theorem 3.1 remains valid for bilateral weighted shifts acting on $\ell_{p}(\mathbb{Z}), 1 \leq p<\infty$.

\subsection{The unilateral weighted backward shift.}

Here we consider a unilateral weighted shift $U$ on the complex Hilbert space of sequences $\ell_{2}$, that is defined by

$$
U e_{n}=w_{n} e_{n-1}, n \geq 2 \quad U e_{1}=0
$$

where $n$ runs through the set of all positive integers $\mathbb{N},\left\{e_{n}: n \in \mathbb{N}\right\}$ is the natural orthonormal basis of $\ell_{2}$, and $\left(w_{n}\right)$ is a bounded sequence of strictly positive numbers, the sequence of weights. The relevant quantity to describe the spectrum of $U$ is

$$
r_{2}(U)=\liminf _{n \rightarrow \infty}\left(w_{1} \cdots w_{n}\right)^{1 / n}
$$

If $r_{2}(U)>0$ then $U_{p}=\left\{\lambda|\lambda|<r_{2}(U)\right\} \subset \sigma_{p}(U)$ and consists of simple eigenvalues (see [18] Theorem 8).

A similar argument to the one given in Subsection 3.1 provides the following result. 
Theorem 3.3 Let $U$ be an unilateral weighted backward shift with $r_{2}(U)>0$. Then every operator in the commutant of $U$ different from $\lambda I$ has a hypercyclic multiple.

3.3 The adjoint of the Cesàro operator on the sequences spaces

The Cesàro operator $C: \ell_{p} \rightarrow \ell_{p}(1<p<\infty)$ is given by

$$
C\left(\left(x_{n}\right)\right)=\left(\frac{x_{1}+\cdots+x_{n}}{n}\right)
$$

and has norm equal to $q$, where $1 / p+1 / q=1$.

The adjoint of the Cesàro operator $C^{\star}: \ell_{q} \rightarrow \ell_{q}$ is given by

$$
C^{\star} e_{n}=\frac{e_{1}+\cdots+e_{n}}{n} .
$$

Let us denote $U_{q}=\{\lambda \in \mathbb{C}:|\lambda-q / 2|<q / 2\}$, and let us consider the sequences $\hat{x}(\lambda)=\left(x_{n}(\lambda)\right)_{n=1}^{\infty}$, where

$$
x_{1}(\lambda)=1 \quad \text { and } \quad x_{n}(\lambda)=\prod_{j=1}^{n-1}\left(1-\frac{1}{j \lambda}\right) \quad n>1 .
$$

Then $\hat{x}(\lambda) \in \operatorname{Ker}\left(C^{\star}-\lambda I\right)$ for $\lambda \in U_{q}$ and $U_{q}$ consists of simple eigenvalues of $C^{\star}$ (see [8] for proper references).

Note that that $x_{n}(1 / k) \neq 0$ if and only if $n \leq k$, and that the sequence $(1 / k)$ is contained in $U_{q}$. Hence $\operatorname{span}\left\{\operatorname{Ker}\left(C^{\star}-\lambda I\right): \lambda \in U_{q}\right\}$ is dense in $\ell_{q}$. Moreover it is not difficult to show that the map $\hat{x}: \lambda \in U_{q} \rightarrow \hat{x}(\lambda) \in \ell_{q}$ is analytic.

Therefore the conditions of Theorem 2.1 are fully satisfied by $C^{\star}$, and as in the previous examples we conclude that every operator in the commutant of $C$ different from $\lambda I$ has a hypercyclic multiple.

Remarks 3.4 The Cesàro operator $C$ is also bounded in the space $c_{0}$ of sequences converging to 0 . In this case the adjoint $C^{\star}: \ell_{1} \rightarrow \ell_{1}$ satisfies the same conditions that $C^{\star}$ on $\ell_{q}$ with $U_{1}=\{\lambda \in \mathbb{C}:|\lambda-1 / 2|<1 / 2\}$. Thus it satisfies the hypothesis of Theorem 2.1. 


\subsection{The Cesàro operator on spaces of integrable functions}

The Cesàro operator $C: L_{p}(0,1) \rightarrow: L_{p}(0,1)(1<p<\infty)$ is given by

$$
C f(x)=\frac{1}{x} \int_{0}^{1} f(s) d s
$$

and has norm equal to $q$, where $1 / p+1 / q=1$. Moreover, it was proved by Leibowitz (see [12]) that the point spectrum of $C$ is the set

$$
U_{p}=\{\lambda \in \mathbb{C}:|\lambda-q / 2|<q / 2\}=\{\lambda \in \mathbb{C}: \operatorname{Re}(1 / \lambda)>1 / q\},
$$

that $U_{p}$ consists of simple eigenvalues of $C$, and that for every $\lambda \in U_{p}$, the function $g_{\lambda}(x)=x^{-1+1 / \lambda}$ satisfies $C g_{\lambda}=\lambda g_{\lambda}$.

Obviously, the function $\lambda \in U_{p} \rightarrow g_{\lambda} \in L_{p}(0,1)$ is analytic. Moreover, since $f_{1 / n}(x)=x^{n-1}$ for all $n \geq 1$, the set $\operatorname{span}\left\{f_{\lambda}: \lambda \in U_{p}\right\}$ is dense in $L_{p}(0,1)$. Therefore the conditions of Theorem 2.1 are fully satisfied by $C$, and we can conclude that every operator in the commutant of $C$ different from $\lambda I$ has a hypercyclic multiple.

\subsection{The adjoint of the Cesàro operator on Hardy spaces of analytic functions}

The Cesàro operator acting on the space $H(\mathbb{D})$ of analytic functions on the unit disk $\mathbb{D}$ is defined formally by

$$
C f(z)=\sum_{n=0}^{\infty}\left(\frac{1}{n+1} \sum_{k=0}^{n} a_{k}\right) z^{n}
$$

where $f(z)=\sum_{k=0}^{\infty} a_{k} z^{k}$. The operator $C$ is bounded on several Banach spaces of analytic functions on $\mathbb{D}$. Boundedness of $C$ on the Hardy space $H^{p}(\mathbb{D})$ was proved by Hardy for $p=2$, and by A. Siskakis for $1 \leq p<\infty$ ([19]). It is not bounded acting on $H^{\infty}(\mathbb{D})$. For further examples we refer to [14].

We will need the following properties provided by Persson [14, Theorem B].

Theorem 3.5 Let $1<p<\infty$, and let $C$ be the Cesàro operator acting on the Hardy space $H^{p}(\mathbb{D})$.

(1) $\sigma(C)=\{\lambda \in \mathbb{C}:|\lambda-p / 2| \leq p / 2\}$

(2) For $|\lambda-p / 2|<p / 2$, the range of $C-\lambda I$ is a closed subspace of $H^{p}(\mathbb{D})$ of codimension 1 . 
Let us denote $U_{p}:=\{\lambda \in \mathbb{C}:|\lambda-p / 2|<p / 2\}=\{\lambda \in \mathbb{C}: \operatorname{Re}(1 / \lambda)>1 / p\}$. It follows from part (2) of Theorem 3.5 that each $\lambda \in U_{p}$ is a simple eigenvalue of the adjoint operator $C^{\star}$.

For each $\lambda \in U_{p}$ we consider the function $f_{\lambda}$ defined on the unit disk $\mathbb{D}$ by

$$
f_{\lambda}(z)=(1-z)^{\frac{1}{\lambda}-1}
$$

Proposition 3.6 Let $1<p<\infty$ and let $q$ such that $1 / p+1 / q=1$.

(1) For each $\lambda \in U_{p}, f_{\lambda} \in H^{q}(\mathbb{D})$.

(2) The function $\lambda \in U_{p} \rightarrow f_{\lambda} \in H^{q}(\mathbb{D})$ is analytic.

Proof. Once we establish (1), part (2) is immediate.

As usual, we identify $H^{q}(\mathbb{D})$ with the subspace of functions in $L^{q}(\mathbb{T})$ whose Fourier coefficients satisfy $\hat{f}(n)=0$ for $n<0$.

To prove (1), it is enough to note that $\lambda \in U_{p}$ implies $\operatorname{Re}(1 / \lambda)-1>-1 / q$; therefore $\left|f_{\lambda}(z)\right|^{q} \leq$ const $|1-z|^{\alpha}$ with $\alpha>-1$, hence $f_{\lambda} \in H^{q}(\mathbb{D})$.

Recall that for $1<p<\infty, H^{p}(\mathbb{D})$ is a complemented subspace of $L^{p}(\mathbb{T})$. As a consequence, the dual space $H^{p}(\mathbb{D})^{\star}$ is isomorphic to $H^{q}(\mathbb{D})$. In fact, for every $G \in H^{p}(\mathbb{D})^{\star}$ there exists $g \in H^{q}(\mathbb{D})$ such that for each $f \in H^{p}(\mathbb{D})$,

$$
\langle G, f\rangle=\frac{1}{2 \pi} \int_{\mathbb{T}} \overline{g(t)} f(t) d t .
$$

In this way, given the Cesàro operator $C$ acting on $H^{p}(\mathbb{D})(1<p<\infty)$, we can see $C^{\star}$ as an operator acting on $H^{q}(\mathbb{D})$.

With this identification, each $f \in H^{p}(\mathbb{D})$ has the form $f(\theta)=\sum_{n=0}^{\infty} a_{n} e^{i n \theta}$, the action of $C$ is given by

$$
(C f)(\theta)=\sum_{n=0}^{\infty} \frac{1}{n+1}\left(\sum_{k=0}^{n} a_{k}\right) e^{i n \theta},
$$

and it easy to check that the action of the adjoint $C^{\star}$ on the elements $e^{i n \theta}$ of $H^{q}(\mathbb{D})$ is given by $C^{\star} e^{i n \theta}=(n+1)^{-1} \sum_{k=0}^{n} e^{i k \theta}$.

Therefore, if $\sum_{n=0}^{\infty} b_{n} e^{i n \theta} \in H^{q}(\mathbb{D})$, then

$$
C^{\star}\left(\sum_{n=0}^{\infty} b_{n} e^{i n \theta}\right)=\sum_{n=0}^{\infty} \frac{b_{n}}{n+1}\left(\sum_{k=0}^{n} e^{i k \theta}\right)=\sum_{k=0}^{\infty}\left(\sum_{n=k}^{\infty} \frac{b_{n}}{n+1}\right) e^{i k \theta} .
$$

Proposition 3.7 As an operator acting on $H^{q}(\mathbb{D})$, $C^{\star}$ satisfies $C^{\star} f_{\lambda}=\lambda f_{\lambda}$ for each $\lambda \in U_{p}$. 
Proof. It is easy to check that for each $\lambda \in U_{p} \backslash\{1 / n: n \in \mathbb{N}\}$, the derivatives of $f_{\lambda}$ satisfy

$$
\frac{f_{\lambda}^{(n)}(0)}{n !}=\prod_{j=1}^{n}\left(1-\frac{1}{j \lambda}\right) .
$$

Proceeding as in the proof of Theorem 2 in [5], we get $C^{\star} f_{\lambda}=\lambda f_{\lambda}$.

The case $\lambda \in\{1 / n: n \in \mathbb{N}\}$ is easy. In fact, denoting $F_{n}$ the subspace generated by $\left\{e^{i k \theta}: 0 \leq k \leq n\right\}$, we have that $C^{\star} F_{n} \subset F_{n}$ for $n \in \mathbb{N} \cup\{0\}$, hence $\left\{f_{1 / n}: n \in \mathbb{N}\right\}$ generates a dense subspace of $H^{q}(\mathbb{D})$.

As a consequence we obtain the following result.

Theorem 3.8 Let $C$ be the Cesàro operator acting on $H^{p}(\mathbb{D}), 1<p<\infty$. Then every operator in the commutant of $C^{\star}$ different from $\lambda I$ has a hypercyclic multiple.

\subsection{Composition operators without a "hypercyclic commutant"}

Here we analyze some examples of operators $T$ which do not satisfy the hypothesis of Theorem 2.1, and also fail to have a hypercyclic commutant.

(a) In the absence of an open set contained in the point spectrum, an operator does not satisfy the hypothesis of Theorem 2.1. This is the case for the following example studied by Godefroy and Shapiro (see [7] Section 3.7).

Let $B$ be a compact unilateral weighted backward shift on the sequence space $\ell^{p}, 1 \leq p<\infty$. By a result of Salas (see [15]), $I+B$ is hypercyclic. However $\lambda B$ is hypercyclic for no $\lambda \in \mathbb{C}$ because $\lambda B$ is quasinilpotent and the orbit of each vector is bounded.

(b) Next we consider some examples of operators for which the eigenvalues are not simple, and they do not have hypercyclic commutant.

Let $\varphi$ denote a linear fractional selfmap of the unit disc, and let $C_{\varphi}$ be the composition operator induced by $\varphi$ acting on the Hardy space $H^{2}(\mathbb{D})$. Its action is given by $C_{\varphi} f=f \circ \varphi$. The linear dynamics of such composition operators was studied by Bourdon and Shapiro ([4]). A comprehensive treatment on dynamics of composition operators is carried out in the books [4] and [16].

Theorem 3.9 Let $\varphi$ be a linear fractional self map of the unit disk, and let $C_{\varphi}$ denote the associated composition operator. Then there exists an operator $T$ in the commutant of $C_{\varphi}$ such that $\lambda T$ is hypercyclic for no $\lambda \in \mathbb{C}$. 
Proof. The only cases which is necessary to check are those in which $C_{\varphi}$ is hypercyclic, namely, when $\varphi$ is a hyperbolic or parabolic automorphism (see [16] Chapter 7). In these cases, the point spectrum of $C_{\varphi}$ contains the point 1 (see [6] Chapter 7, or [17] Theorems 4.1.1 (ii), 4.1.2 and 4.1.3).

Let $f$ be a non-zero function such that $C_{\varphi} f=f$. The the analytic Toeplitz operator $T_{f}$ commutes with $C_{\varphi}$ and $T_{f}$ has not a hypercyclic multiple because $\sigma_{p}\left(T_{f}^{\star}\right)$ is non-empty.

\section{Concluding remarks}

To understand the influence of hypercyclic operators in linear dynamics it would be desirable to characterize operators with a hypercyclic commutant.

Let us denote by $H$ an infinite dimensional complex Hilbert space. Using the ideas of D. A. Herrero in [10] (see also [13] Section 4) together with Theorem 2.1 we obtain the following result.

Corollary 4.1 Let us denote by $\mathcal{R}(H)$ the set of hypercyclic operators acting on $H$ with a hypercyclic commutant. Then the closure of $\mathcal{R}(H)$ in the norm topology is equal to the norm-closure of the set of all hypercyclic operators.

Thus, although the set of operators with a hypercyclic commutant is invariant under similarity, it does not seem that this class can be characterized in spectral terms. To understand the nature of this phenomenon we suggest to analyze the following question.

Problem 4.2 Let $T$ be a unilateral (respectively bilateral) weighted backward shift with weight sequence $\left\{w_{n}\right\}$ defined on $\ell^{p}$, (respectively $\left.\ell_{p}(\mathbb{Z})\right) 1 \leq p<\infty$.

Is it be possible to characterize when $T$ has a hypercyclic commutant in terms of the sequence $\left\{w_{n}\right\}$ ?

The Cesàro operator is also bounded on other spaces of analytic functions on the unit disc (see [14]) such as the Bergman spaces $L_{a}^{p}, p>1$, weighted Bergman spaces $L_{a}^{p, \alpha}$ and Dirichlet spaces $D^{2, \alpha}$. From the results of Persson it follows that the adjoint $C^{\star}$ of the Cesàro operator on these spaces has an open set of set of simple eigenvalues. However we do not know a good representation on the dual space of such spaces.

Conjecture 4.3 The adjoint $C^{\star}$ of the Cesàro operator $C$ acting on $L_{a}^{p}$, $L_{a}^{p, \alpha}$ or $D^{2, \alpha}$ has a hypercyclic commutant. 


\section{References}

[1] P. Aiena. Fredholm and local spectral theory with applications to multipliers, Kluwer Acad. Publ., Dordrecht, 2004.

[2] Frédéric Bayart and Étienne Matheron. Dynamics of linear operators, volume 179 of Cambridge Tracts in Mathematics. Cambridge University Press, Cambridge, 2009.

[3] Teresa Bermúdez and Vivien G. Miller. On operators $T$ such that $f(T)$ is hypercyclic. Integral Equations Operator Theory, 37(3):332-340, 2000.

[4] Paul S. Bourdon and Joel H. Shapiro. Cyclic phenomena for composition operators. Mem. Amer. Math. Soc. 125, 1997.

[5] Arlen Brown, P. R. Halmos, and A. L. Shields. Cesàro operators. Acta Sci. Math. (Szeged), 26:125-137, 1965.

[6] Carl C. Cowen and Barbara D. MacCluer. Composition operators on spaces of analytic functions. Studies in Advanced Mathematics. CRC Press, Boca Raton, FL, 1995.

[7] Gilles Godefroy and Joel H. Shapiro. Operators with dense, invariant, cyclic vector manifolds. J. Funct. Anal., 98(2):229-269, 1991.

[8] Manuel González. The fine spectrum of the Cesàro operator in $l_{p}(1<p<\infty)$. Arch. Math. (Basel), 44(4):355-358, 1985.

[9] Karl-G. Grosse-Erdmann and Alfredo Peris Manguillot. Linear chaos. Universitext. Springer, London, 2011.

[10] Domingo A. Herrero. Limits of hypercyclic and supercyclic operators. J. Funct. Anal., 99(1):179-190, 1991.

[11] Gerd Herzog and Christoph Schmoeger. On operators $T$ such that $f(T)$ is hypercyclic. Studia Math., 108(3):209-216, 1994.

[12] G. M. Leibowitz. Spectra of finite range Cesàro operators. Acta Sci. Math. (Szeged), 35:27-29, 1973.

[13] Vladimir Müller. On the Salas theorem and hypercyclicity of $f(T)$. Integral Equations Operator Theory, 67(3):439-448, 2010.

[14] Anna-Maria Persson. On the spectrum of the Cesàro operator on spaces of analytic functions. J. Math. Anal. Appl., 340(2):1180-1203, 2008.

[15] Héctor N. Salas. Hypercyclic weighted shifts. Trans. Amer. Math. Soc., 347(3):993-1004, 1995.

[16] Joel H. Shapiro. Composition operators and classical function theory. Universitext: Tracts in Mathematics. Springer-Verlag, New York, 1993. 
[17] Joel H. Shapiro. Strongly compact algebras associated with composition operators. New York J. Math., 18:849-875, 2012.

[18] Allen L. Shields. Weighted shift operators and analytic function theory. In Topics in operator theory, pages 49-128. Math. Surveys, No. 13. Amer. Math. Soc., Providence, R.I., 1974.

[19] Aristomenis G. Siskakis. Composition semigroups and the Cesàro operator on $H^{p}$. J. London Math. Soc. (2), 36(1):153-164, 1987. 